\title{
ROBUSTNESS EVALUATION OF A MULTIVARIABLE FRACTIONAL ORDER PI CONTROLLER FOR TIME DELAY PROCESSES
}

\author{
Cristina I. Muresan, * Eva H. Dulf, ${ }^{*}$ and Clara Ionescu*
}

\begin{abstract}
Fractional order calculus is currently experiencing a wide application in controlling different types of processes. The focus has been directed towards the design of robust controllers for single-inputsingle-output systems, with very few tuning examples for the multivariable ones. The purpose of the present paper is to describe a simple and effective method for designing a multivariable fractional order PI controller for a multivariable time delay system. The main idea of the tuning procedure is extended from the singleinput-single-output case with the simple use of a steady state decoupling technique. The design method is based on performance specifications that ensure a certain settling time and on a gain robustness condition. The solution is found by using an iterative procedure. The case study presented demonstrates the efficiency of the proposed control design, the closed loop system behaving robustly to significant gain variations ranging $\pm 30 \%$. Also, the robustness of the fractional order multivariable controller is tested against time delay variations.
\end{abstract}

\section{Key Words}

Multivariable fractional order PI, Smith predictor, decoupling, robustness

\section{Introduction}

Fractional order controllers have been in the spotlight of the control engineering community in the last decade [1]-[4], having been successfully used in controlling both integerorder and fractional order (FO) systems, with the main target of enhancing the robustness and performance of the control systems [2], [5]. The increasing interest towards FO controllers, especially the FO $P I^{\mu} D^{\lambda}$, is based on their potential to improve the control performance [6]-[8], due to the two supplementary tuning variables involved, $\mu$ and $\lambda$.

\footnotetext{
* Technical University of Cluj-Napoca, Department of Automatic Control, Str. Memorandumului, no. 28, 400114 Cluj-Napoca, Romania; e-mail: \{Cristina.Pop, Eva.Dulf\}@aut.utcluj.ro, Claramihaela.Ionescu@ugent.be

Recommended by Prof. K.K. Tan
}

(DOI: 10.2316/Journal.201.2014.2.201-2507)
However, the majority of the works conducted in the field of FO controllers deal with plants of the form of single input single output (SISO) systems, with only a few results obtained for multivariable FO PID controllers design [9], [10]. Previous techniques for designing the multivariable FO PID controllers consist in an approach similar to the SISO case. In [10], the novel method proposed by the authors is based on an $H_{\infty}$ problem with a controller structure constraint, while the controller parameters are optimized to achieve both user-specified robust stability and performance, the controller obtained being tested for controlling systems with multiple delays [11].

The present paper proposes a different approach in the design of a multivariable FO PI controller for systems with multiple time delays. The proposed method is simpler and offers significant robustness against gain uncertainties. The actual design of the multivariable FO PI controller is in fact an extension of the SISO case. Such an approach is possible, since prior to the design of the controller the multivariable system is firstly decoupled using a steady state decoupling technique. For the new decoupled process, several SISO FO PI controllers are designed and later the multivariable FO PI controller is computed.

The design of the SISO FO PI controllers is based on a robustness specification for gain uncertainties and a settling time performance criteria, imposed using the gain crossover frequency. The general approach towards determining the controller parameters is by using the somewhat time-consuming optimization routines. The approach taken in this paper is based on a simpler method that employs an iterative procedure to determine the tuning parameters. Since the design focuses on multivariable time delay processes, the control structure used consists in a Smith predictor, which facilitates the design of the multivariable FO PI controller [11].

The paper is structured into four parts. Immediately after the Introduction, the proposed control method is presented, including the decoupling procedure, the multivariable FO PI controller design in a Smith predictor structure and the implementation procedure of the controller 
obtained. Section 3 contains a case study to exemplify the tuning procedure described in Section 2. The design is presented step by step, while the last part of the section presents the simulation results that demonstrate the efficiency of the proposed control method. Since the control structure used is a multivariable Smith predictor, time delay variations are also considered in the simulations. It is shown that the multivariable FO PI controller behaves robustly even under this assumptions. The last part of the paper contains the concluding remarks.

\section{Multivariable FO Controller Design}

The transfer function matrix for a general $m \times m$ square process may be written as:

$$
G_{p}(s)=\left[\begin{array}{ccc}
g_{11} e^{-\tau_{11} s} & \ldots & g_{1 m} e^{-\tau_{1 m} s} \\
: & : & : \\
g_{m 1} e^{-\tau_{m 1} s} & \cdots & g_{m m} e^{-\tau_{m m} s}
\end{array}\right]
$$

where $g_{i j}$ represent first order transfer functions from the $j$ th input to the $i$ th output [11], [12]. The model of the multivariable process, under nominal conditions, is equal to $G_{p}(s)$ :

$$
G_{m}(s)=G_{p}(s)
$$

The steady state gain matrix of the model $G_{m}(s)$ is given by:

$$
G_{m}(s=0)=\left[\begin{array}{ccc}
g_{110} & \ldots & g_{1 m 0} \\
: & : & : \\
g_{m 10} & \ldots & g_{m m 0}
\end{array}\right]
$$

The steady state decoupling of the process is achieved using the inverse of the steady state gain matrix, $G_{m}^{\#}$ [11]-[13]:

$$
G_{D}(s)=G_{m}(s) \cdot G_{m}^{\#}=\left[\begin{array}{ccc}
g_{d 11} & \ldots & g_{d 1 m} \\
: & : & \ldots \\
g_{d m 1} & \ldots & g_{d m m}
\end{array}\right]
$$

All elements in the decoupled process transfer function matrix $G_{D}(s)$ are weighted sums of the original transfer functions of $G_{m}(s), g_{i j} e^{-\tau_{i j} s}$. Due to the steady state decoupling, all elements of the main diagonal in $G_{D}(s)$ will have a unitary value, while all non-diagonal elements will have zero values. As a consequence, the non-diagonal terms will not be considered in the design of the controller [11].

The design of the FO PI controller would results in a tedious work due to the complicated form of the diagonal elements. To facilitate the design of the controller, these diagonal terms are further approximated [11], [12], [14] with simpler transfer functions. The approximated transfer functions of the decoupled process $G_{D}(s)$ are denoted as:

$$
g_{d i i}(s) \approx g_{d i i}^{*}(s)
$$

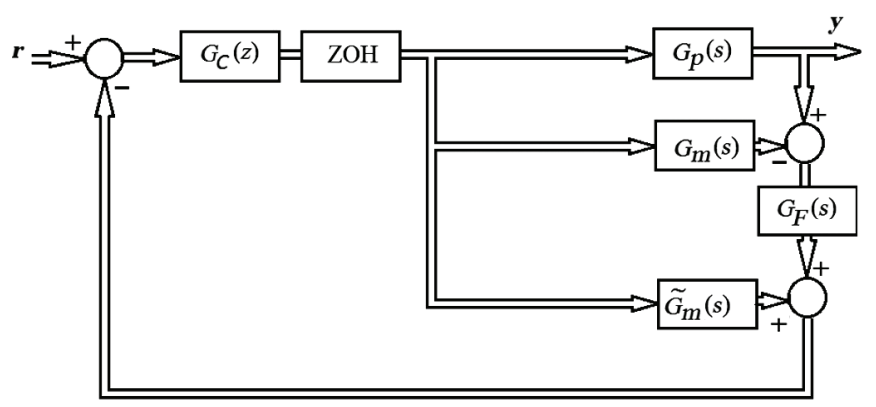

Figure 1. Proposed control structure.

For each of these approximations in (5), individual FO PI controllers are designed:

$$
H_{F O-P I}(s)=k_{p}\left(1+\frac{k_{i}}{s^{\mu}}\right)
$$

where $\mu \in(0,1)$ is the FO. The design of the controller consists in determining the controller parameters, $k_{p}$ and $k_{i}$, as well as the FO $\mu$. Since the control structure consists in a Smith predictor, the tuning of the parameters is done based on the delay free part of the transfer function in (5), denoted as $g_{d i i}^{*}(s)[11]$.

The control structure proposed in this paper consists of the Smith predictor, with multivariable FO PI primary controller [11]. The control structure used is given in Fig. 1, where $\tilde{G}_{m}(s)$ is the process model without the time delays and $G_{F}(s)$ are feedback filters added to improve robustness to time delay variations [14]-[15].

The tuning of the FO PI controller is done independently for each $\widetilde{\sigma}_{d i i}^{*}(s)$, by imposing a gain crossover frequency, to establish the settling time of the closed loop [11], [16], and by imposing robustness to gain changes. Based on the imposed gain crossover frequency, the following equation is obtained:

$$
\left|H_{d}\left(j \omega_{g c}\right)\right|=1
$$

where $\omega_{g c}$ is the gain crossover frequency. Equation (7) may be rewritten as:

$$
\left|H_{F O-P I}\left(j \omega_{g c}\right) \cdot \underset{g_{d i i}^{*}}{\approx}\left(j \omega_{g c}\right)\right|=1
$$

If $L$ is the imaginary part of ${\underset{g}{d i i}}^{*}\left(j \omega_{g c}\right)$ and $K$ is the real part of $\underset{g_{d i i}^{*}}{\approx}\left(j \omega_{g c}\right)$, then (8) yields:

$$
\left|\frac{1}{K+j L}\right|_{\omega_{g c}}\left|k_{p}\left[1+k_{i} \omega_{g c}^{-\mu}\left(\cos \frac{\pi \mu}{2}-j \sin \frac{\pi \mu}{2}\right)\right]\right|=1
$$

leading to:

$$
k_{p}=\sqrt{\frac{L^{2}+K^{2}}{1+2 k_{i} \omega_{g c}^{-\mu} \cos \frac{\pi \mu}{2}+k_{i}^{2} \omega_{g c}^{-2 \mu}}}
$$


The gain robustness condition may be written as:

$$
\frac{d\left(\angle H_{d}\left(j \omega_{g c}\right)\right)}{d \omega_{g c}}=0
$$

The phase of $H_{d}\left(j \omega_{g c}\right)$ is given by:

$$
\angle H_{d}\left(j \omega_{g c}\right)=a \tan \left(-\frac{k_{i} \omega_{g c}^{-\mu} \sin \frac{\pi \mu}{2}}{1+k_{i} \omega_{g c}^{-\mu} \cos \frac{\pi \mu}{2}}\right)-a \tan \left(\frac{L}{K}\right)
$$

Taking the derivative of (12) with respect to $\omega_{g c}$, yields:

$$
\frac{\mu k_{i} \omega_{g c}^{-\mu-1} \sin \frac{\pi \mu}{2}}{1+2 k_{i} \omega_{g c}^{-\mu} \cos \frac{\pi \mu}{2}+k_{i}^{2} \omega_{g c}^{-2 \mu}}-\frac{\dot{L} K-L \dot{K}}{L^{2}+K^{2}}=0
$$

Using (10) and (13), $k_{p}$ and $k_{i}$ can be uniquely determined, for a given FO $\mu$ [11].

The tuning algorithm may be described as:

for $\mu=0: 1$

compute $k_{i}$ using (10)

compute $k_{p}$ using (10)

end

$\operatorname{plot}\left(\mu,\left[k_{i} k_{p}\right]\right)$

select $\mu_{b}$ such that the curves for $k_{i}$ and $k_{p}$ intersect

compute final values for $k_{p}$ and $k_{i}$ using $\mu_{b}$

The continuous form of the final controller in Fig. 1 is then obtained using [11], [14]:

$G_{C}(s)=G_{m}^{\#}\left(\begin{array}{cccc}H_{F O-P I_{1}}(s) & 0 & \ldots & 0 \\ 0 & H_{F O-P I_{2}}(s) & \ldots & 0 \\ \ldots & \ldots & \ldots & 0 \\ 0 & 0 & \ldots H_{F O-P I_{m}}(s)\end{array}\right)$

The FO PI in $(6)$, with $\mu \in(0,1)$, is implemented as:

$$
H_{F O-P I}(s)=k_{p}\left(1+\frac{k_{i} s^{1-\mu}}{s}\right)
$$

to ensure the effect of an integer order integrator, both at high and low frequencies [17].

Next, the FO PI controllers given by (15) are discretized using the fifth order recursive Tustin discretization method [18], with the sampling period $T$ :

$$
s^{\mu}=\left(\frac{2}{T}\right)^{\mu} \frac{A\left(z^{-1}, \mu\right)}{A\left(z^{-1},-\mu\right)}
$$

and,

$$
\begin{aligned}
A\left(z^{-1}, \mu\right)= & -\frac{\mu}{5} z^{-5}+\frac{\mu^{2}}{5} z^{-4}-\left(\frac{\mu}{3}+\frac{\mu^{3}}{15}\right) z^{-3} \\
& +\frac{2}{5} \mu^{2} z^{-2}-\mu z^{-1}+1
\end{aligned}
$$

Using the discretization method in (16), the $G_{C}(z)$ controller in Fig. 1 is obtained.

\section{Case Study: ${ }^{13} \mathrm{C}$ Isotope Separation Column}

To exemplify the algorithm described in Section 2, a case study consisting in the control of the secondary processes of a cryogenic carbon isotope separation column is considered [11], [13]:

$$
\left(\begin{array}{l}
y_{1} \\
y_{2} \\
y_{3}
\end{array}\right)=\left(\begin{array}{llc}
g_{11}(s) e^{-\tau_{11} s} & g_{12}(s) e^{-\tau_{12} s} & 0 \\
g_{21}(s) e^{-\tau_{21} s} & g_{22}(s) e^{-\tau_{22} s} & g_{23}(s) \\
g_{31}(s) e^{-\tau_{31} s} & g_{32}(s) e^{-\tau_{32} s} & g_{33}(s)
\end{array}\right)\left(\begin{array}{l}
u_{1} \\
u_{2} \\
u_{3}
\end{array}\right)
$$

with $g_{11}(s)=\frac{-0.1111}{s^{2}+1.094 s+0.08423}, \quad g_{23}(s)=\frac{-1.104}{s+0.1176}$

$$
\begin{aligned}
& g_{12}(s)=\frac{0.1152}{s^{2}+1.211 s+0.2021}, g_{33}(s)=\frac{8.457}{s+0.9851} \\
& g_{21}(s)=\frac{-0.001731}{s^{2}+0.1343 s+0.001961} \\
& g_{22}(s)=\frac{0.003846}{s^{2}+0.1547 s+0.004357} \\
& g_{31}(s)=\frac{-0.009918}{s^{2}+1.056 s+0.07036} \\
& g_{32}(s)=\frac{0.006288}{s^{2}+1.085 s+0.09851}
\end{aligned}
$$

The steady state gain matrix is [11], [13]:

$$
G_{m}(s=0)=\left[\begin{array}{ccc}
-1.318 & 0.569 & 0 \\
-0.882 & 0.882 & -9.386 \\
-0.140 & 0.063 & 8.585
\end{array}\right]
$$

with its inverse being equal to [11]:

$$
G_{m}^{\#}=\left[\begin{array}{ccc}
-1.432 & 0.856 & 0.936 \\
-1.558 & 1.982 & 2.167 \\
-0.011 & -6.7 \cdot 10^{-4} & 0.115
\end{array}\right]
$$

The time delays matrix of the MIMO system in (18) is given in Table 1, with values given in minutes.

Table 1

Time Delay Matrix of the Secondary Processes (min)

\begin{tabular}{|r|r|r|c|}
\hline & $U_{1}$ & $U_{2}$ & $U_{3}$ \\
\hline$y_{1}$ & 10 & 32 & 0 \\
\hline$y_{2}$ & 10 & 8 & 0 \\
\hline$y_{3}$ & 18 & 35 & 0 \\
\hline
\end{tabular}


The decoupled process transfer function matrix can then be computed using (4), resulting in the following diagonal terms [11]:

$$
\begin{aligned}
g_{d 11}= & -1.432 \cdot g_{11}(s) e^{-\tau_{11} s}-1.558 \cdot g_{12}(s) e^{-\tau_{12} s} \\
g_{d 22}= & 0.856 \cdot g_{21}(s) e^{-\tau_{21} s}+1.982 \cdot g_{22}(s) e^{-\tau_{22} s} \\
& +6.7 \cdot 10^{-4} \cdot g_{23}(s) \\
g_{d 33}= & 0.936 \cdot g_{31}(s) e^{-\tau_{31} s}+2.167 \cdot g_{32}(s) e^{-\tau_{32} s} \\
& +0.115 \cdot g_{33}(s)
\end{aligned}
$$

The diagonal terms in (21) are approximated with the following transfer functions [11]:

$$
\begin{aligned}
& g_{d 11}^{*}(s)=\frac{0.022274 \cdot e^{-6 s}}{\left(s^{2}+0.1044 s+0.02223\right)} \\
& g_{d 22}^{*}(s)=\frac{0.0045918 \cdot e^{-8 s}}{\left(s^{2}+0.12 s+0.004592\right)} \\
& g_{d 33}^{*}(s)=\frac{1.0565}{(s+1.057)}
\end{aligned}
$$

Next, for each of the delay free transfer functions in (22), the FO PI controllers are designed by imposing the robustness condition in (13), as well as the required gain crossover frequencies:

$$
\begin{aligned}
& \omega_{g c 1}=0.01 \\
& \omega_{g c 2}=0.001 \\
& \omega_{g c 3}=0.5
\end{aligned}
$$

Using the algorithm described in Section 2, for each of the diagonal terms, the following curves are obtained, as presented in Figs. 2-4.

The parameters of the FO PI controller $-k_{i}, k_{p}$ and $\mu$ - are uniquely determined at the intersection point of the two curves in each of the Figs. 2-4:

$$
\begin{aligned}
& \mu_{1}=0.364, \quad k_{p 1}=0.35 \text { and } k_{i 1}=0.363 \\
& \mu_{2}=0.276, \quad k_{p 2}=0.323 \text { and } k_{i 2}=0.32 \\
& \mu_{3}=0.925, \quad k_{p 3}=0.596 \text { and } k_{i 3}=0.765
\end{aligned}
$$

Using the previously determined values in (24) and replacing them in (15), the transfer functions of the FO PI controllers are obtained as:

$$
\begin{aligned}
& H_{F O-P I_{1}}(s)=0.35\left(1+\frac{0.363 s^{1-0.364}}{s}\right) \\
& H_{F O-P I_{2}}(s)=0.323\left(1+\frac{0.32 s^{1-0.276}}{s}\right) \\
& H_{F O-P I_{3}}(s)=0.596\left(1+\frac{0.765 s^{1-0.925}}{s}\right)
\end{aligned}
$$

The continuous form of the final controller in (14) is thus computed as:

$$
G_{C}(s)=G_{m}^{\#}\left(\begin{array}{ccc}
H_{F O-P I_{1}}(s) & 0 & 0 \\
0 & H_{F O-P I_{2}}(s) & 0 \\
0 & 0 & H_{F O-P I_{3}}(s)
\end{array}\right)
$$

with the matrix $G_{m}^{\#}$ given in (20).

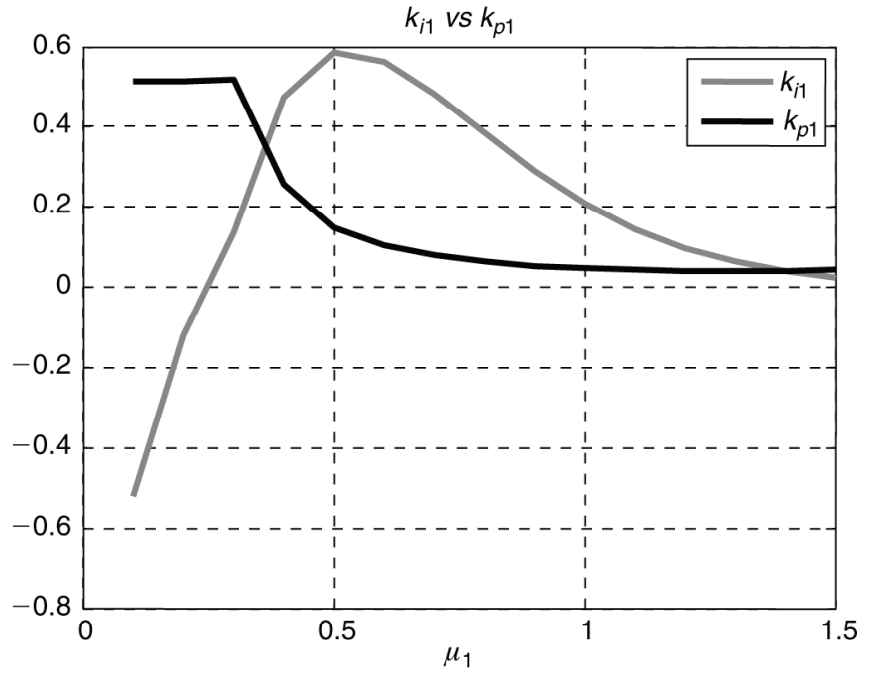

Figure 2. FO PI controller parameters as a function of $\mu$, for $g_{d 11}^{*}(s)$.

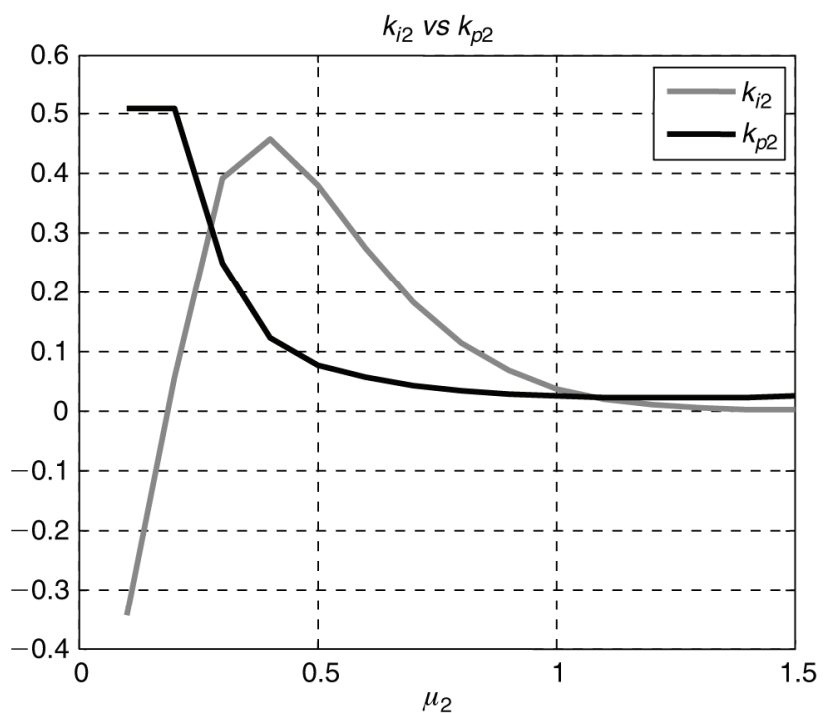

Figure 3. FO PI controller parameters as a function of $\mu$, for $g_{d 22}^{*}(s)$.

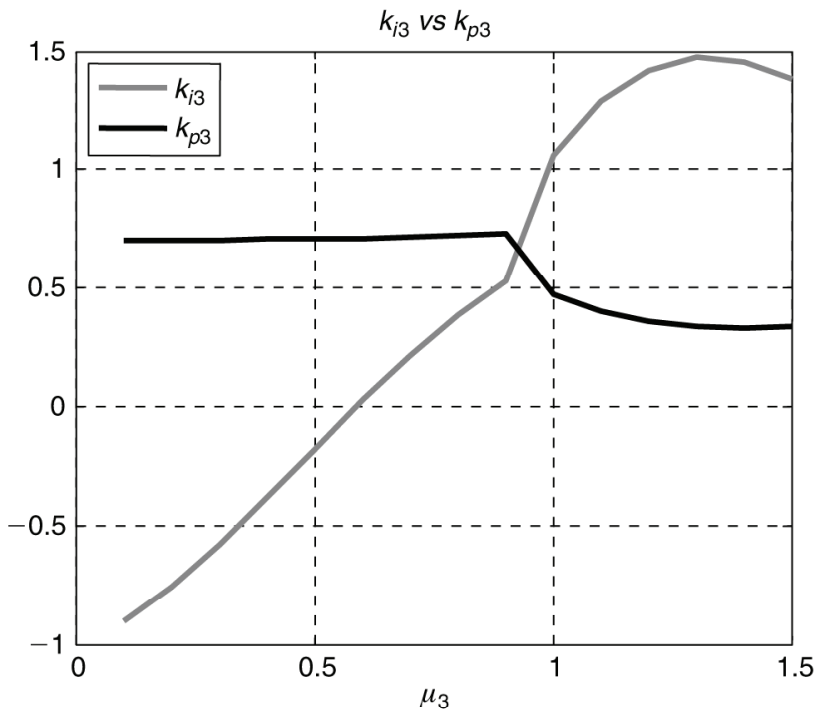

Figure 4. FO PI controller parameters as a function of $\mu$, for $g_{d 33}^{*}(s)$. 


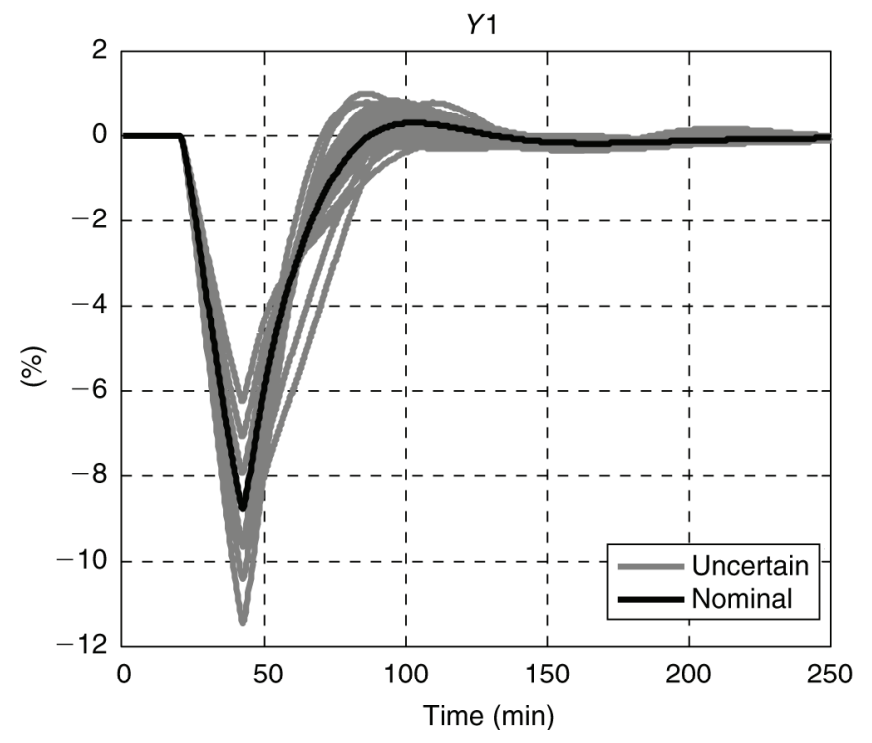

Figure 5. Output $y_{1}$ evolution considering a step change in the $y_{2}$ reference and open loop gain variations.

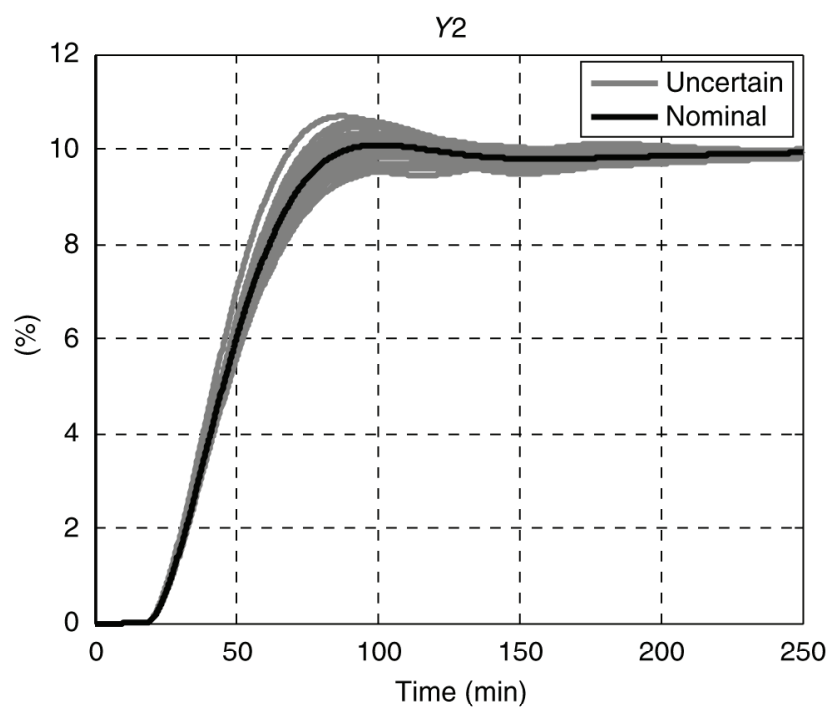

Figure 6. Output $y_{2}$ evolution considering a step change in its reference signal and open loop gain variations.

Using (16) and (17), the discrete forms of the FO PI controllers in (25) are obtained [11]:

$$
\begin{aligned}
s^{1-0.364} & =\left(\frac{2}{T}\right)^{1-0.364} \frac{A\left(z^{-1}, 1-0.364\right)}{A\left(z^{-1},-(1-0.364)\right)} \\
s^{1-0.276} & =\left(\frac{2}{T}\right)^{1-0.276} \frac{A\left(z^{-1}, 1-0.276\right)}{A\left(z^{-1},-(1-0.276)\right)} \\
s^{1-0.925} & =\left(\frac{2}{T}\right)^{1-0.925} \frac{A\left(z^{-1}, 1-0.925\right)}{A\left(z^{-1},-(1-0.925)\right)}
\end{aligned}
$$

with a sampling time $T=0.3 \mathrm{~min}$.

Figures 5-7 present the three outputs evolution, considering a step change in the second output reference signal, while Figs. 8-10 show the evolution of the three inputs.

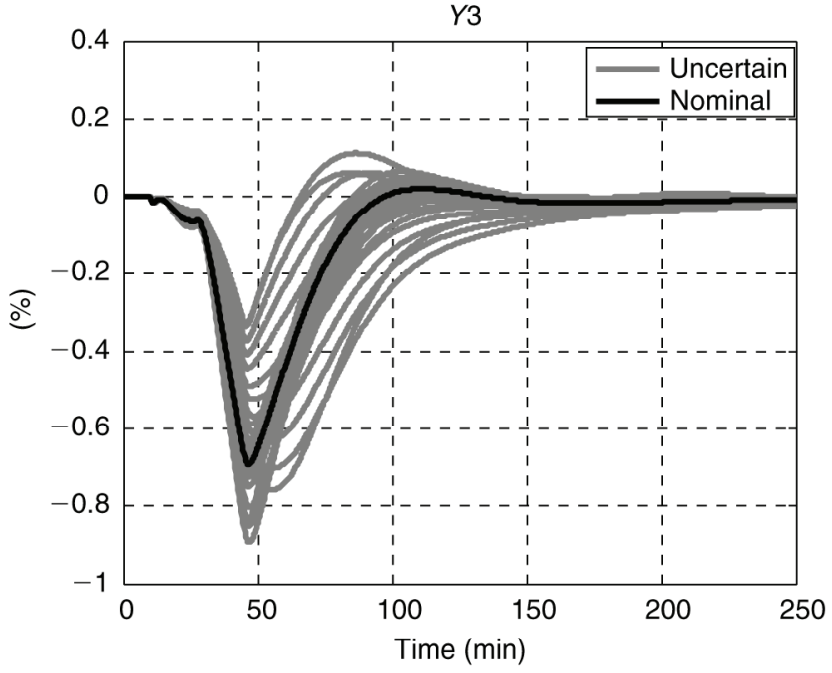

Figure 7. Output $y_{3}$ evolution considering a step change in the $y_{2}$ reference and open loop gain variations.

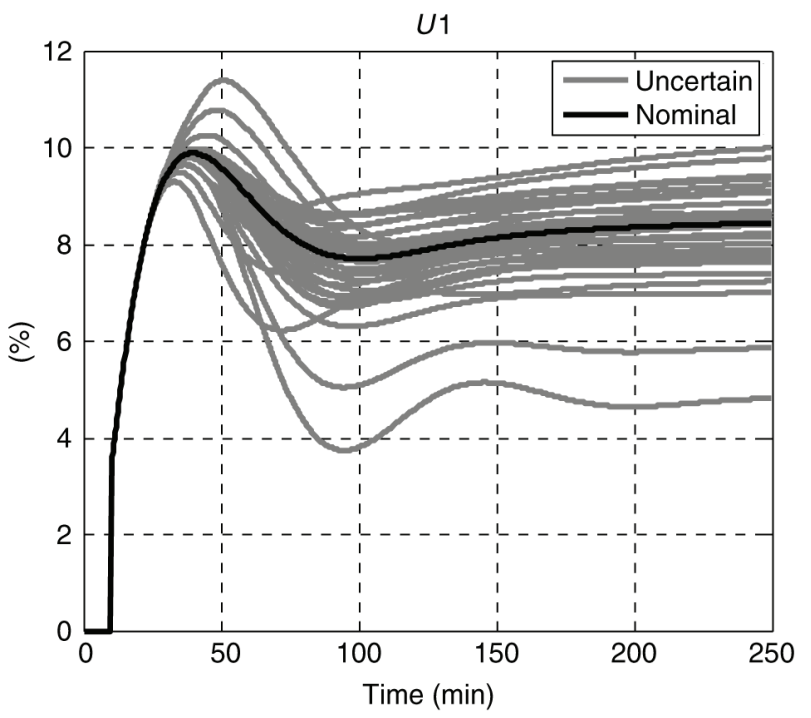

Figure 8. Input $u_{1}$ evolution considering a step change in the $y_{2}$ reference and open loop gain variations.

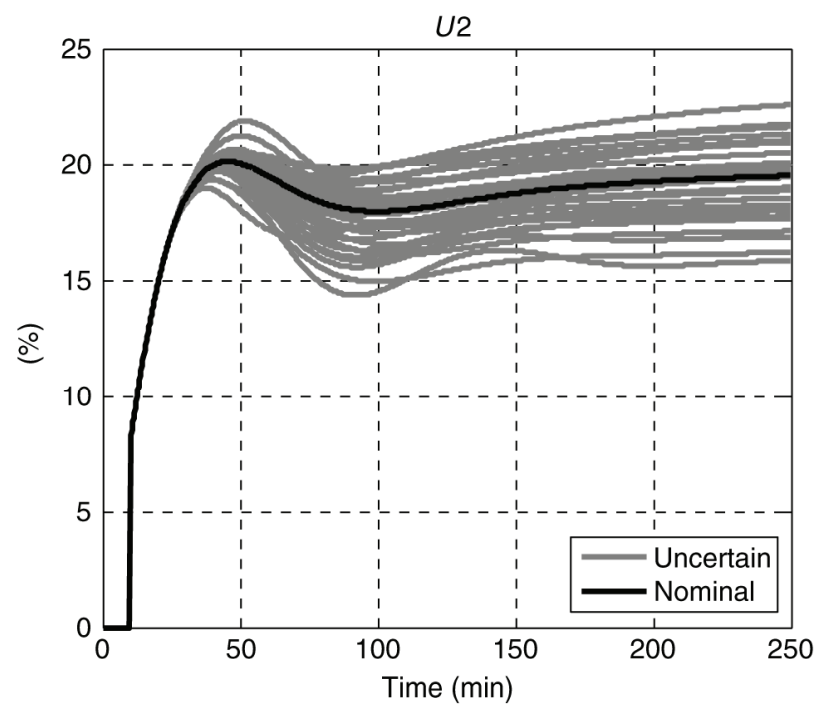

Figure 9. Input $u_{2}$ evolution considering a step change in the $y_{2}$ reference and open loop gain variations. 


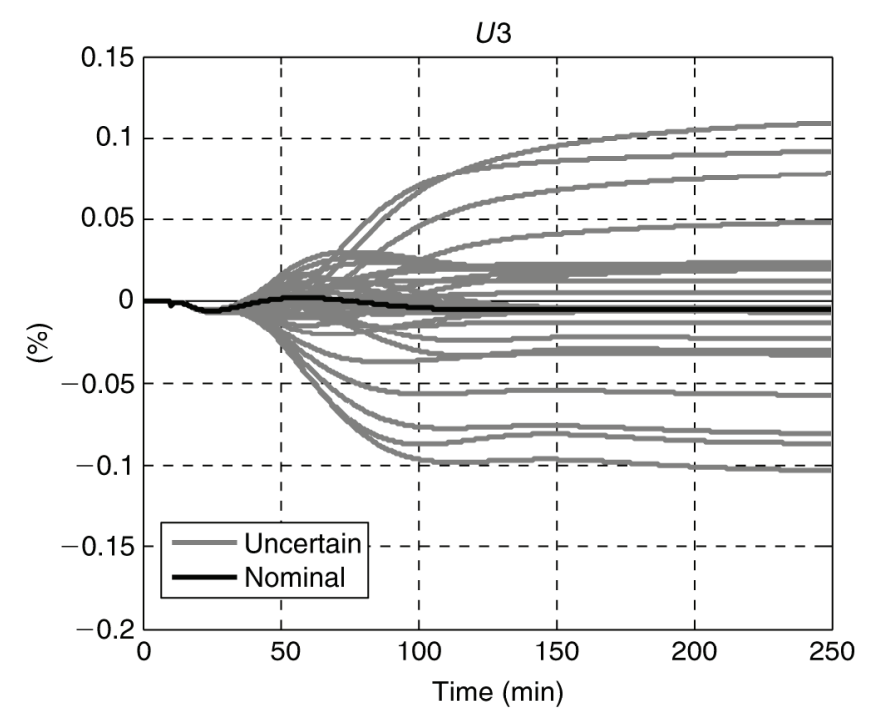

Figure 10. Input $u_{3}$ evolution considering a step change in the $y_{2}$ reference and open loop gain variations.

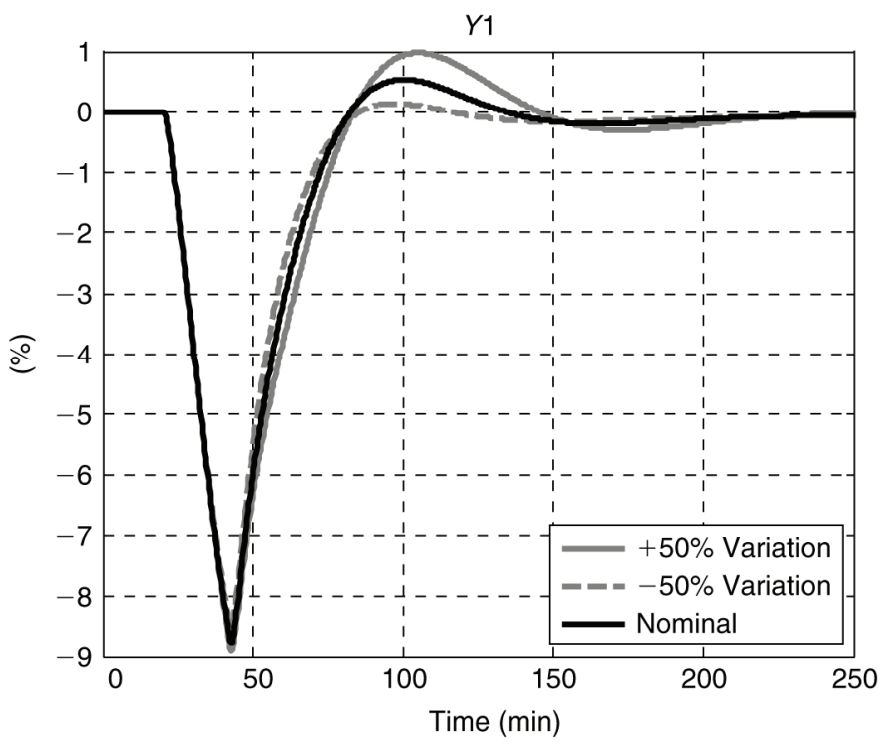

Figure 11. Output $y_{1}$ evolution considering a step change in the $y_{2}$ reference and time delay variations.

The nominal case scenario, considering $G_{p}(s)=G_{m}(s)$, as well as situations considering $\pm 30 \%$ gain variations are presented in the figures. The results obtained clearly indicate that the robustness specification regarding gain uncertainties is attained, with the maximum overshoot being less than $10 \%$.

The main problem regarding the use of the Smith predictor structure is related to the possible instability of the closed loop system against time delay variations. Such a problem has also been considered by testing the designed multivariable FO PI controller against significant time delay uncertainties of $+50 \%$, as well as $-50 \%$, respectively. The simulations results, considering the three outputs evolution are given in Figs. 11-13.

The same case scenario is considered, with a step change of $10 \%$ in the reference of the second output. In the nominal case, the overshoot is of $4 \%$, while the settling time is below $150 \mathrm{~min}$. Considering the time delay variations, the overshoot is increased to a maximum of

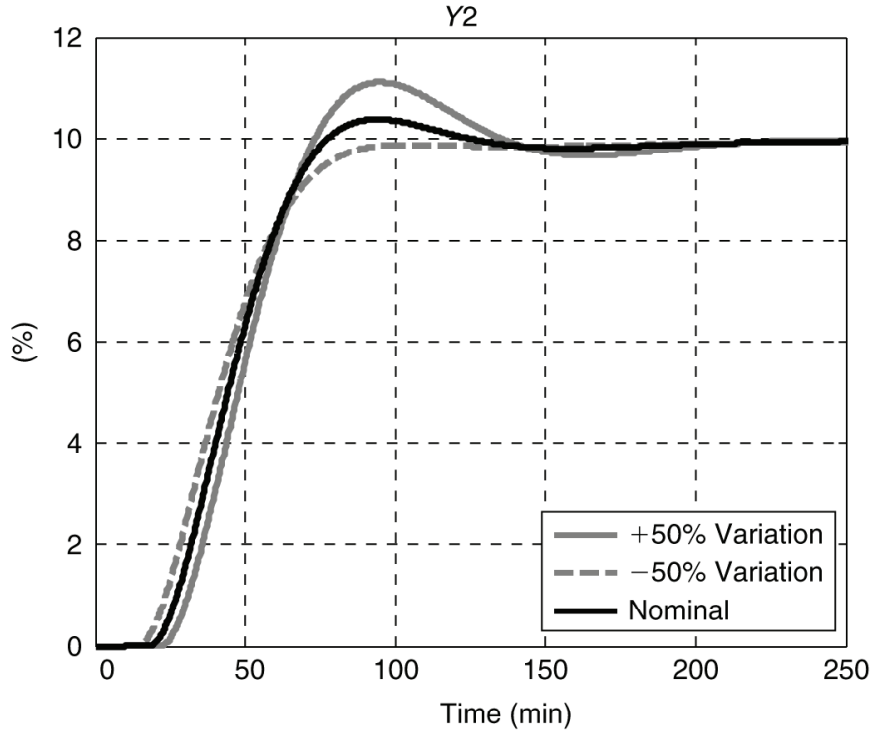

Figure 12. Output $y_{2}$ evolution considering a step change in its reference and time delay variations.

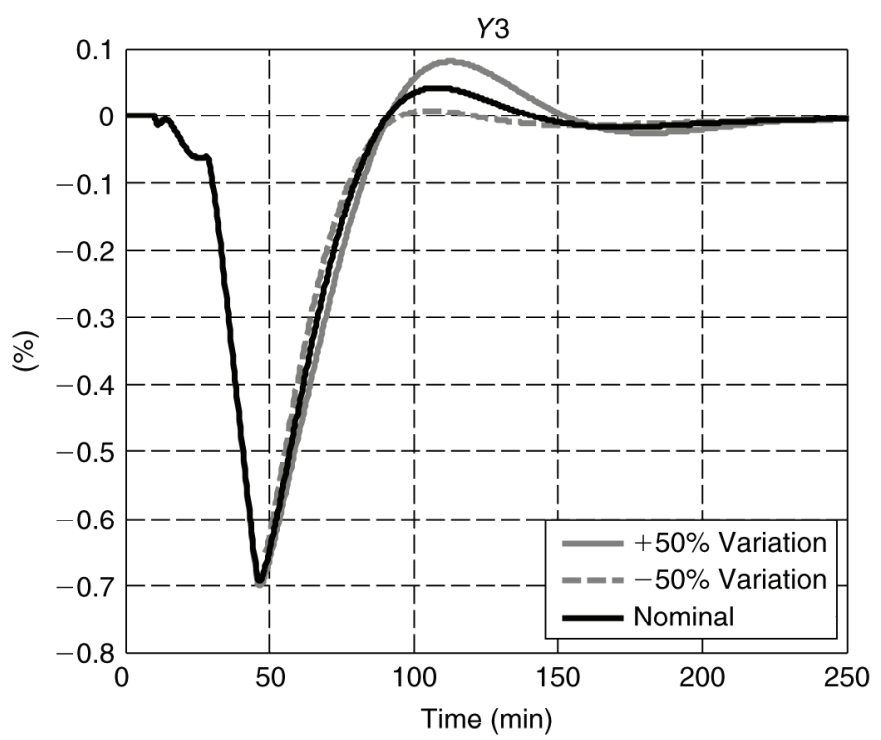

Figure 13. Output $y_{3}$ evolution considering a step change in the $y_{2}$ reference and time delay variations.

$10 \%$, while the settling time remains below $150 \mathrm{~min}$. The simulation results show that the designed multivariable FO PI controller can meet the specifications imposed even in the presence of time delay variations.

\section{Conclusion}

The main purpose of the paper was to provide a simple and efficient method for designing multivariable FO PI controllers with increased robustness against gain uncertainties. The case study presented demonstrates that the proposed method can achieve good results both under nominal conditions and in situations of process gain variations. Since the use of the Smith predictor may lead to possible instability of the closed loop system, in situations that involve time delay variations, the multivariable FO PI controller was additionally tested for checking the ability to cope with such situations. The simulation results presented show that the designed controller is not only robust 
to open loop gain variations, as expected from the tuning procedure, but also to time delay uncertainties.

\section{Acknowledgement}

This work was supported by a grant of the Romanian National Authority for Scientific Research, CNDIUEFISCDI, project number PN-II-PT-PCCA-2011-3.2$0591155 / 2012$.

\section{References}

[1] A. Oustaloup, B. Mathieu, and P. Lanusse, The CRONE control of resonant plants: application to a flexible transmission, European Journal of Control, 1, 1995, 113-121.

[2] I. Podlubny, Fractional-order systems and $\mathrm{PI}^{\lambda} \mathrm{D}^{\mu}$-controllers, IEEE Transactions on Automation and Control, 44, 1999, 208-214.

[3] D. Xue and Y. Chen, A comparative introduction of four fractional order controllers, Proc. 4th IEEE World Congress on Intelligent Control and Automation, Shanghai, China, 2002, 3228-3235.

[4] Y. Luo, Y.Q. Chen, C.Y. Wang, and Y.G. Pi, Tuning fractional order proportional integral controllers for fractional order systems, Journal of Process Control, 20, 2010, 823-831.

[5] A. Oustaloup, La Commande CRONE: Commande Robuste d'Ordre Non Entier (Paris, France: Hermes, 1991).

[6] C.A. Monje, B.M. Vinagre, Y.Q. Chen, and V. Feliu, Proposals for fractional PID-tuning, Proc. First IFAC Symposium on Fractional Differentiation and its Application (FDA04), Bordeaux, France, 2004.

[7] D. Xue, C. Zhao, and Y.Q. Chen, Fractional order PID control a DC-motor with elastic shaft: a case study, Proc. 2006 American Control Conference, Minnesota, 2006, 3182-3187.

[8] A. Oustaloup, J. Sabatier, and P. Lanusse, From fractional robustness to CRONE control, Fractional Calculus and Applied Analysis, 2, 1999, 1-30.

[9] X. Song, Y.Q. Chen, I. Tejado, and B.M. Vinagre, Multivariable fractional order PID controller design via LMI approach, Proc. 18th IFAC World Congress, Milano, Italy, 2011.

[10] S. Chenikher, S. Abdelmalek, and M. Sedraoui, Control of uncertainly multi-variable system with fractional PID, Proc. 2012 16th IEEE Mediterranean Electrotechnical Conference (MELECON), Yasmine Hammamet, Tunisia, 2012, 1079-1082.

[11] C.I. Muresan, E.H. Dulf, and C. Ionescu, Multivariable fractional order PI controller for time delay processes, International Conference on Engineering and Applied Science, DOI: 10.2316/P.2012.785-029, 27-29 December 2012, Colombo, Sri Lanka.

[12] J. Chen, Z.F. He, and X. Qi, A new control method for MIMO first order time delay non-square systems, Journal of Process Control, 21, 2011, 538-546.

[13] C.I. Pop. C.M. Ionescu, and R. De Keyser, Time delay compensation for the secondary processes in a multivariable carbon isotope separation unit, Chemical Engineering Science, 80, 2012, 205-218.

[14] C.I. Pop, R. De Keyser, and C.M. Ionescu, A simplified control method for multivariable stable nonsquare systems with multiple time delays, Proc. 19th Mediterranean Conference on Control and Automation, Corfu, Greece, 2011, 382-387.

[15] R.A. Seshagiri and M. Chidambaram, Smith delay compensator for multivariable non-square system with multiple time delays, Computers and Chemical Engineering, 30(8), 2006, 1243-1255.

[16] M. Sam Fadali, Digital control engineering: Analysis and design (Burlington, MA: Academic Press, 2009).

[17] C.A. Monje, Y. Chen, B.M. Vinagre, D. Xue, and V. Feliu, Fractional-order systems and controls: fundamentals and applications (London, UK: Springer, 2010).

[18] Y.Q. Chen and K.L. Moore, Discretization schemes for fractional-order differentiators and integrators, IEEE Transactions of Circuits and Systems - I: Fundamental Theory and Applications, 49(3), 2002, 363-367.

\section{Biographies}

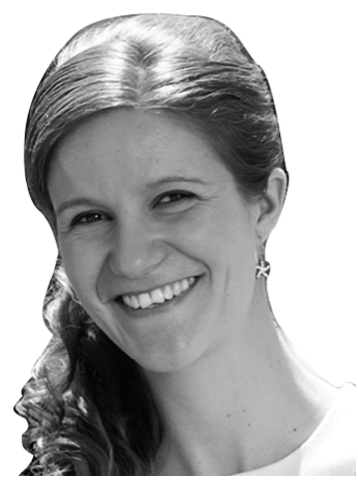

Cristina I. Muresan received the degree in control systems in 2007, and the Ph.D. in 2011 from Technical University of Cluj-Napoca, Cluj-Napoca, Romania. She is currently lecturer at the Technical University of Cluj-Napoca, Automation Department, Romania. Since 2007, she has published over 30 papers and book chapters, amongst which 2 have been awarded by the Romanian government. She has been and currently is involved in 4 research grants, all dealing with multivariable and fractional order control. Her research interests include modern control strategies, such as predictive algorithms, robust nonlinear control, fractional order control, time delay compensation methods and multivariable systems.

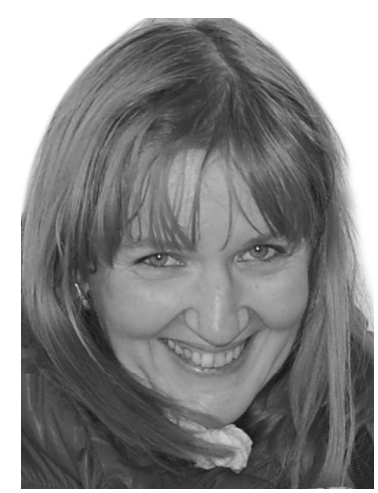

Eva H. Dulf received her B.Eng. degree in automation from Technical University of Cluj-Napoca, Romania, in 1997, and her M.Sc. and Ph.D. degrees from the same university in 1998 and 2006, respectively. In November 1998, she joined Technical University of Cluj-Napoca as teaching assistant and she is now associate professor. Current research interests include various areas in process control, including mathematical modelling, controller design, optimization methods and practical implementation. She has published more than 100 technical journal papers and conference talks, and she is a member of IEEE.

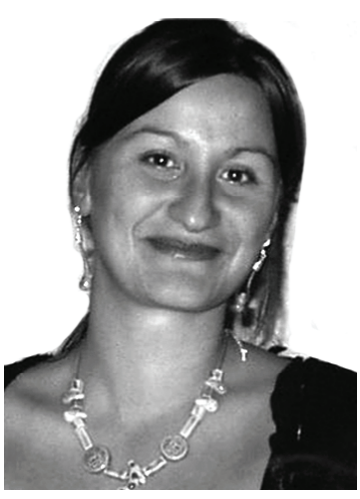

Clara Ionescu (S'06-M'09) was born in 1979, Cimpulung, Romania. She received the M.Sc. degree in industrial informatics and automation from "Dunarea de Jos" University, Galati, Romania, in 2003. She obtained the Ph.D. degree at Ghent University, Gent, Belgium in 2009, on identification of human respiratory system by means of fractional order models. She is involved in several international projects, with both industrial and biomedical applications, for identification and control. She has about 100 peer-reviewed publications in journals, conferences and books, being actively involved in promoting the concept of fractality in biological systems and their applications to medicine. 